\title{
Critical Sensing Range for Mobile Heterogeneous Camera Sensor Networks
}

\author{
Yitao Hu, Xinbing Wang, Xiaoying Gan \\ Department of Electronic Engineering, Shanghai Jiao Tong University, Shanghai, China \\ Email: \{oldjack, xwang8, ganxiaoying\}@ sjtu.edu.cn
}

\begin{abstract}
In camera sensor networks (CSNs), full view coverage, in which any direction of any point in the operational region is covered by at least one camera sensor, is of great significance since image shot at the frontal viewpoint considerably increases the possibility to recognize the object. However, finding the critical condition to achieve full view coverage in mobile heterogeneous CSNs remains an open question. In this paper, we analyze both the static and mobile random deployed camera sensor networks. A centralized parameter - equivalent sensing radius (ESR) - is defined to evaluate the critical requirement for asymptotic full view coverage in heterogeneous CSNs. We derive the critical sensing range for full view coverage under static model, 2-dimensional random walk mobility model, 1dimensional random walk mobility model and random rotating model. We then discuss the impact of various mobility patterns on sensing energy consumption and study the power-delay tradeoff and show that random walk mobility model can decrease the sensing energy consumption under certain delay tolerance. To our knowledge, our work is the very first that derive the critical condition to achieve full view coverage in mobile heterogeneous CSNs.
\end{abstract}

\section{INTRODUCTION}

Coverage, a critical performance metric in Wireless Sensor Networks (WSNs), is used to measure how well a field is monitored by sensors within the field. It is significant in many applications, such as security surveillance, intrusion detection in battlefield or military zone and so forth. Recently, Camera Sensor Networks (CSNs) have attracted an increasing amount of attention, since the image or video provided by camera sensors can considerably enrich the information of the monitored region. Such networks have prospective applications including traffic avoidance, environmental monitoring, and industrial process control [1]. However, the camera sensors do not possess omnidirectional sensing ability as traditional ones. On the contrary, they can only sense within an angle of view, beyond which it is unable to capture any information, which leads to new problems and requirements for networks.

There are many investigations for networks of traditional sensors. In [2], Kumar studied the asymptotic k-coverage in a mostly sleepy stationary sensor network, and in [3], Srikant et al. considered the failure probability and obtained the necessary and sufficient condition to achieve asymptotic full coverage. In[4], [5] and [6], the authors mainly focused on the barrier coverage problems with traditional sensors separately. Besides, in [7] and [8], the authors studied deployment scheme to achieve multiple coverage and connectivity. And in [9], Bai and Kumar proposed a deployment pattern to achieve both coverage and connectivity.

However, compared to the relative mature study on the coverage in traditional sensor networks, studies on the coverage of CSNs are quite limited. In [10], Wang and Cao first proposed a novel concept in the judgement of coverage in CSNs, called full view coverage. An object is full view covered if its viewed direction is always closely enough to its facing direction, wherever it actually faces. Since the frontal image could provide a higher probability for computer recognition systems to successfully recognize an object [11], it is of great significance to develop a CSN being able to achieve full view coverage.

To construct such networks, it is essential to understand the required conditions for full view coverage, e.g. sensing radius, angle of view, or deployment density. In [10], Wang and Cao provided a sufficient condition under random and uniform deployment, and a critical (i.e. both necessary and sufficient) condition under triangle lattice based deployment. And in [12], the authors analyzed the necessary and sufficient condition to achieve full view coverage separately. Other existing works on CSNs are mainly concerned with full view barrier coverage as in [13] and [14], which suggests the critical condition to achieve full view area coverage in mobile camera sensor network still remains an open problem.

In this paper, we concentrate on the critical condition [15] of full view coverage under uniform deployment. We consider asymptotic coverage for mathematical convenience, which means that the total number of cameras approaches to infinity. The coverage problem of an unit square is converted into the coverage of a dense grid in it, which is a common method in the analysis of area coverage [2]. Following this route, we derive the critical condition of full view coverage under uniform deployment.

We also investigate the impact of mobility. In [16], Liu proposed that mobility could improve coverage performance, since it could reduce the detection time of intruders. Saipulla and Liu in [17] considered the limited mobility for barrier coverage which distinguish their work. In [18], Chen studied sweep coverage for mobile sensors. Besides, mobility was found to increase the capacity [19] and help security [20] in ad-hoc networks. All of these works reveal the significance of analysis of mobility. In this paper, we study the critical sensing range for full view coverage under three different mobile patterns and compare them with the static model to verify their advantages. 
Moreover, we take heterogeneity of camera sensors into consideration. It is intuitive to treat CSNs as a heterogeneous network, since camera sensors may come from different manufacturers and thus have different sensing parameters, or the sensing capability of cameras will decline as time passes by or under different obstruction of terrains. To deal with heterogeneous cameras, we divide them into different groups according to their sensing parameters as the cases in [21] and [22]. The equivalent sensing radius (ESR) are defined later to help analyzing in static and mobile condition separately. This index summarizes different sensing parameters of all the cameras, and therefore represents the overall requirements for cameras in CSNs.

Our main contributions are highlighted as follows.

- We provide the critical condition of full view coverage under four different static or mobile patterns, which could help the engineers to design the CSN according to certain engineering requirements by balancing coverage performance and sensing energy consumption.

- We define the ESR for static and mobile camera sensor network, which could provide intrinsic understanding of heterogeneous camera sensor network. For static model, $r=\sqrt{\sum_{y=1}^{u} c_{y} \frac{\phi_{y}}{2 \pi} r_{y}^{2}}$. For 2-dimensional random walk mobility model and 1-dimensional random walk mobility model, $r=\sum_{y=1}^{u} c_{y} \frac{\phi_{y}}{2 \pi} r_{y}$. And for random rotating mobility model, $r=\sqrt{\sum_{y=1}^{u} c_{y}\left(2-\left(1-\frac{\phi_{y}}{2 \pi}\right)^{2}\right) r_{y}^{2}}$.

- Compared with static model, 2-dimensional random walk mobility and 1-dimensional random walk mobility reduces the sensing energy consumption by the order $\Theta\left(\frac{\log n+\log (\log n)}{n \theta}\right)^{1}$, at the expense of $\Theta(1)$ delay under uniform deployment.

The remainder of the paper is organized as follows. Basic models and definitions are described in Section II. In Section III, we study the static model and derive the ESR to achieve full view coverage. We study 2-dimensional random walk mobility model, 1-dimensional random walk mobility model and random rotating model and derive the corresponding ESR in Section IV. In Section V, we study the power-delay tradeoff. In Section VI, we conclude the paper and discuss about the future work.

\section{NotATiOnS AND Model}

In this section we present the sensing and deployment model of the camera sensors used in our paper, introduce our mobility patterns, and describe several performance measures, like the definition and meaning of full view coverage and equivalent sensing range, to assess the coverage performance of static and mobile heterogeneous CSNs.

\footnotetext{
${ }^{1}$ The following asymptotic notations are used throughout this paper. Given non-negative functions $f(n)$ and $g(n)$ :

1) $f(n)=\Theta(g(n))$ means that for two constants $0<c_{1}<c_{2}$, $c_{1} g(n) \leq f(n) \leq c_{2} g(n)$ for sufficiently large $n$.

2) $f(n) \sim g(n)$ means that $\lim _{n \rightarrow+\infty} \frac{f(n)}{g(n)}=1$.

3) $f(n)=o(g(n))$ means that $\lim _{n \rightarrow+\infty} \frac{f(n)}{g(n)}=0$.
}

\section{A. Deployment Scheme and Sensing Model}

In this paper, we assume the operational region of the sensor network is an unit torus square, such that we don't need to consider strategy when sensors reach the edge of the area, and focus on the general cases. Actually coverage problem near the boundaries differs significantly from general situations, but it is currently beyond the scope of this paper.

We assume in the operational region $n$ sensors are randomly and uniformly deployed, independent of each other, which is widely recognized as proper estimations for randomly distributed sensors. The random strategy is favored in the situation that the operational region is inimical and hostile, or that it is expensive and difficult to place sensors by human or programmed robots. Under such circumstance, wireless sensors might be sprinkled from aircrafts, delivered by artillery shell, rocket, missile or thrown from a ship, instead of being placed by human or programmed robots.

A camera sensor $S$ can sense perfectly in a sector of radius $r$ and angle $\phi$, but will not sense outside the sector. Without confusion, $S$ also denotes the location of the sensor. The angular bisector of $\phi$ is recognized as orientation of $S$, denoted by $\vec{f}$. This model is commonly used in literature [23] and [24], called binary sector model. Further, since the quality of information provided by a camera is sensitive to its viewpoint, there are other two essential directions to be considered. The direction towards which a point $P$ faces is called its facing direction, denoted by $\vec{p}$. The vector $\overrightarrow{P S}$ is called viewed direction of the object, which reflects the viewpoint of sensor $S$. Figure 1 illustrates these directions which will be useful in subsequent discussion.

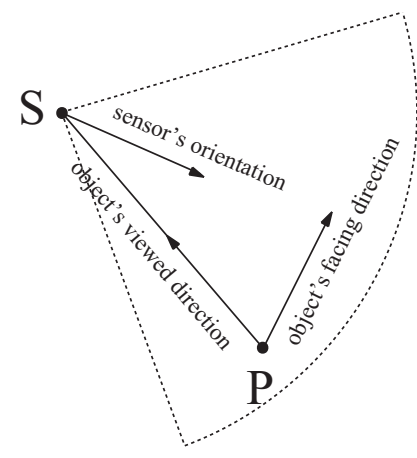

Fig. 1. For sensor $S$ and point $P$, the orientation, viewed direction and facing direction are depicted respectively.

We consider heterogeneous sensors similar to [21]. To describe sensors of different qualities, we partition sensors to $u$ groups $G_{1}, G_{2}, \cdots, G_{u}$, where $u$ is a constant. As the total number of sensors is $n$, each group $G_{y}(y=1,2, \cdots, u)$ has $n_{y}=c_{y} n$ sensors, where $c_{y}$ is a constant invariant to $n$. Clearly, $c_{y}$ satisfies $0<c_{y}<1$ and $\sum_{y=1}^{u} c_{y}=1$. All sensors in group $G_{y}$ own identical sensing radius $r_{y}$ and angle $\phi_{y}$, but either $r_{y} \neq r_{z}$ or $\phi_{y} \neq \phi_{z}$ will hold if $y \neq z(y, z=1,2, \cdots, u)$. We mainly study the asymptotic coverage here, implying that $n$ is a variable approaching to infinity, whereas $r_{y}$ and $\phi_{y}$ are dependent variables of 
$n$, sometimes denoted by $r_{y}(n)$ and $\phi_{y}(n)$. When the total number of sensors $n$ changes, the requirements for $r_{y}(n)$ and $\phi_{y}(n)$ should change along with $n$.

\section{B. Static and Mobility Patterns}

For mobility patterns, we divide the sensing process into time slots with unit length, and sensors can move according to certain mobility patterns in each time slot. When assuming the network works in a large amount of time slots, a single time slot can also be viewed as an instant.

- Static Model: Wherever a sensor locates, its orientation $\vec{f}$ faces towards all possible directions with equal probability. And once a sensor is deployed, neither its orientation $\vec{f}$ nor its location will change, which means that the camera would not steer its lens during the operation.

- 2-Dimensional Random Walk Mobility Model: At the very beginning of each time slot, each sensor uniformly chooses a random direction $\sigma \in[0,2 \pi)$, and then it rotates its facing direction to the chosen one and moves along the direction with a constant velocity $v$ in each time slot and the velocity is $\Theta(1)$.

- 1-Dimensional Random Walk Mobility Model: Sensors are classified into two types of equal quantity, H-nodes and V-nodes. And sensors of each type move horizontally and vertically, respectively. At the very beginning of each time slot, each sensor randomly and uniformly chooses a direction along its moving dimension and travels in the selected direction for a certain distance $D$, a random variable uniformly distributed from 0 to $1 .^{2}$ The velocity of the sensors is not considered, as long as they could reach the destination within the time slot, and remain stationary until the next slot.

- Random Rotating Mobility Model: Cameras can rotate and change their orientation clockwise or counterclockwise. At the very beginning of each time slot, each sensor randomly chooses a rotating direction, i.e. clockwise or counterclockwise, and then rotates an angle $\Psi$, a random variable uniformly distributed between 0 and $2 \pi$. Similarly, the velocity of sensors is ignored.

\section{Performance Measures}

To assess the full view coverage performance in CSNs, the following four definitions are proposed.

1) Definition of $\theta$-view coverage: For a specific facing direction $\vec{p}$ of point $\mathrm{P}$, it achieves $\theta$-view coverage if it is covered by at least one sensor and the angle between $\vec{p}$ and its viewed direction is no more than $\theta$. Here, $\theta \in(0, \pi]$ is a predefined constant parameter called effective angle.

2) Definition of Full View Coverage: For a point $P$, it is full view covered if every possible facing direction $\vec{p}$ of it is $\theta$-view covered. The operational region achieves full view coverage iff every point in it achieve full view coverage.

\footnotetext{
${ }^{2}$ Long distance travel is energy-consuming. And if the sensor can travel beyond the dimension of the operational region (i.e., $D>1$ ), it can always cover the area along its moving dimension which is meaningless.
}

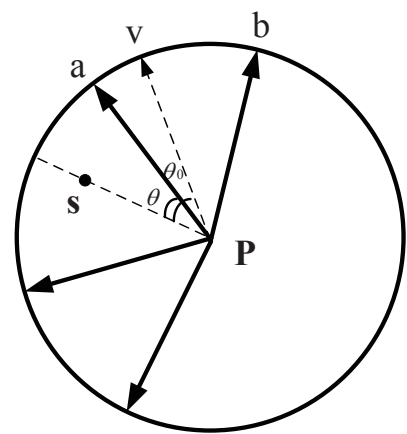

(a)

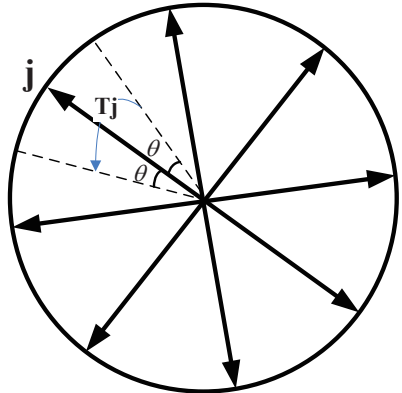

(b)
Fig. 2. (a) shows a set of four nearest directions include $a$ and $b$ in the direction set $\mathbb{K}$, (b) shows the possible area $T_{j}$ to $\theta$-view cover orientation $O_{j}$.

3) Definition of Equivalent Sensing Radius: For heterogeneous camera sensor networks, we define the equivalent sensing radius (ESR) for each static and mobility pattern to analyze the asymptotic full view coverage. The ESR of the heterogeneity CSN for static model is $r=\sqrt{\sum_{y=1}^{u} c_{y} \frac{\phi_{y}}{2 \pi} r_{y}^{2}}$, the ESR for 2-dimensional random walk mobility model or 1-dimensional random walk mobility model is $r_{i}=$ $\sum_{y=1}^{u} c_{y} \frac{\phi_{y}}{2 \pi} r_{y}, i=2 . r . w ., 1 . r . w .$, and the ESR for random rotating mobility model is $r=\sqrt{\sum_{y=1}^{u} c_{y}\left(2-\left(1-\frac{\phi_{y}}{2 \pi}\right)^{2}\right) r_{y}^{2}}$.

In this part, $\frac{\phi_{y}}{2 \pi}$ is viewed as the weight of each sensor's radius. When $\phi=2 \pi$, it is equivalent to a sensor whose sensing range is a circle, and ESR in this case is the same as the ESR for omnidirectional sensors in [21].

4) Definition of Critical ESR: Let $\mathcal{H}$ denotes the event that the operational region is full view covered. Then if

$$
\begin{array}{r}
\lim _{n \rightarrow \infty} \mathbb{P}(\mathcal{H})=1, \text { if } r_{i} \geq c R_{i}(n) \text { for any } c>1 ; \\
\lim _{n \rightarrow \infty} \mathbb{P}(\mathcal{H})<1, \text { if } r_{i} \leq \hat{c} R_{i}(n) \text { for any } 0<\hat{c}<1,
\end{array}
$$

where $R_{i}(n)$ is the critical ESR under four different static and mobile patterns, and $i=$ stat,r.r., 2.r.w., 1.r.w..

\section{Overview of the Geometric Analysis and Preliminaries}

In [21], Wang proves that, the full coverage of a $\sqrt{m} \times$ $\sqrt{m}$ dense grid $\mathbb{M}$ can promise the full coverage of the unit square, when $m=n \log n$, based on THEOREM 4.1 in [2]. We can also prove that the $\theta$-view coverage of a facing direction set $\mathbb{K}$ formed by $k$ directions of a point can promise its full view coverage when $k=n \log n$, and we derive the following theorem.

Theorem 1. For point $P$, if a $k$ facing direction set $\mathbb{K}$ satisfying $k=n \log n$, the $\theta$-view coverage of set $\mathbb{K}$ can promise the full view coverage of $P$ with effective angle $\theta$ when $n$ is large enough.

The proof is available in technical report [25].

Thus we can focus on the $\theta$-view coverage of orientation set $\mathbb{K}$ for the dense gird $\mathbb{M}$ to estimate full view coverage performance of the operational region. 
III. THE CRiticAl SENSING RANGE FOR StATiC CAMERA SENSOR NETWORKS

In this section, we mainly focus on the full view coverage for static camera sensor networks under uniform deployment, and obtain the equivalent sensing range for heterogeneous cameras. We first analyze the equivalent sensing range for dense grid $\mathbb{M}$, then expand it to case of the whole area and derive the following theorem.

Theorem 2. Under the uniform deployment with static model, the critical ESR for mobile heterogenous CSNs to achieve asymptotic full view coverage is

$$
R_{\text {stat }}(n)=\sqrt{\frac{2(\log n+\log \log n)}{n \theta}} .
$$

Let $\mathbb{P}_{i, j, S_{y}}$ denote the probability that orientation $O_{j}$ of point $P_{i}$ is $\theta$-viewed covered by sensor $\mathrm{S}$ in group $G_{y}$. To make $O_{j}$ of set $\mathbb{K} \theta$-viewed covered, at least one sensor should locate in sector $T_{j}$, as shown in Figure $2(\mathrm{~b})$. For sector $T_{j}$, the angular bisector is orientation $j$, with an angle $2 \theta$. Then

$$
\begin{aligned}
\mathbb{P}_{i, j, S_{y}} & =\mathbb{P}\left(S \text { falls in } T_{j}\right) \times \mathbb{P}(S \text { has proper orientation }) \\
& =\frac{2 \theta}{2 \pi} \times \pi r_{y}^{2}(n) \times \frac{\phi_{y}}{2 \pi}=\frac{r_{y}^{2}(n) \phi_{y} \theta}{2 \pi}
\end{aligned}
$$

\section{A. Necessary Condition of Theorem 2}

Let $\mathcal{G}_{r w}(n, r(n))$ denote the network that each point in $\mathbb{M}$ achieves full view coverage when the sensing range is $r(n)$, and we use $P_{f-r w}(n, r(n))$ to represent the probability that $\mathcal{G}_{r w}(n, r(n))$ has at least one point that is not full view covered. Then we derive the following proposition. For simplicity, we say a direction uncovered and not $\theta$-view covered equivalently, and a point uncovered and not full view covered interchangeably.

Proposition 1. In the static heterogeneous CSN, if

$$
r_{\text {stat }}(n)=\sqrt{\frac{2(\log n+\log \log n+\omega(n))}{n \theta}},
$$

$m=n \log n$ and $k=n \log n$, then

$$
\liminf _{n \rightarrow \infty} P_{f-r w}(n, r(n)) \geq e^{-2 \omega}-\frac{\theta}{\pi} e^{-3 \omega},
$$

where $\omega=\lim _{n \rightarrow \infty} \omega(n)$.

proof: To ease the complexity of the proof, we provide the following lemma first.

Lemma 1. Given a variable $x=x(n)$ satisfies $0<x(n)<\frac{1}{2}$, and a variable $y=y(n)>0$, then $(1-x)^{y} \sim e^{-x y}$ if $x^{2} y$ approaches to zero as $n \rightarrow+\infty$.

Using method similar to the proof of LEMMA 1 in [12], we could easily prove Lemma 1 . Then we study the case that $r(n)=\sqrt{\frac{2(\log n+\log \log n+\omega(n))}{n \theta}}$ for a fixed $\omega$. Referring to Bonferroni inequalities, we get

$$
\begin{aligned}
& P_{f-r w}(n, r(n)) \\
& \geq \sum_{P_{i} \in \mathbb{M}} \sum_{O_{j} \in \mathbb{K}} \mathbb{P}\left(\left\{O_{j} \text { of } P_{i} \text { is uncovered }\right\}\right) \\
& -\sum_{P_{i} \in \mathbb{M}} \sum_{O_{j}, O_{h} \in \mathbb{K}}^{O_{j} \neq O_{h}} \mathbb{P}\left(\left\{O_{j} \text { and } O_{h} \text { of } P_{i} \text { are uncovered }\right\}\right) .
\end{aligned}
$$

For the first term of the R.H.S. of Eq. (1)

$$
\begin{aligned}
& \mathbb{P}\left(\left\{O_{j} \text { of } P_{i} \text { is uncovered }\right\}\right) \\
& \geq \prod_{y=1}^{u} \mathbb{P}\left(\left\{O_{j} \text { is uncovered by sensors in } G_{y}\right\}\right) \\
& =\prod_{y=1}^{u}\left(1-\frac{r_{y}^{2}(n) \phi_{y} \theta}{2 \pi}\right),
\end{aligned}
$$

where $\frac{r_{y}^{2}(n) \phi_{y} \theta}{2 \pi}$ represents the probability that orientation $O_{j}$ of point $P_{i}$ is $\theta$-viewed covered by sensor $\mathrm{S}$ in group $G_{y}$, while $c_{y} n$ represents the number of sensors in group $G_{y}$.

Then with Lemma (1) and Eq. (2), we obtain that

$$
\begin{aligned}
\sum_{P_{i} \in \mathbb{M}} \sum_{O_{j} \in \mathbb{K}} \mathbb{P} & \left(\left\{O_{j} \text { of } P_{i} \text { are uncovered }\right)\right. \\
& \sim m k e^{-n \theta \sum_{y=1}^{u} c_{y} \frac{\phi_{y}}{2 \pi} r_{y}^{2}(n)} \\
& =m k e^{-n \theta r_{\text {stat }}^{2}(n)} \\
& =(n \log n)^{2} e^{-2(\log n+\log \log n+\omega)} \\
& =e^{-2 \omega} .
\end{aligned}
$$

For the second term of the R.H.S. of Eq. (1)

$$
\begin{aligned}
& \mathbb{P}\left(\left\{O_{j} \text { and } O_{h} \text { of } P_{i} \text { is uncovered }\right\}\right) \\
& \quad \leq \frac{2 \theta}{2 \pi} \prod_{y=1}^{u}\left(1-\frac{3}{2} \frac{r_{y}^{2}(n) \phi_{y} \theta^{c_{y} n}}{2 \pi}\right) \\
& \quad+\left(1-\frac{2 \theta}{2 \pi}\right) \prod_{y=1}^{u}\left(1-2 \frac{r_{y}^{2}(n) \phi_{y} \theta}{2 \pi}\right),
\end{aligned}
$$

where the two terms on the right side correspond to the cases when $\angle\left(O_{j}, O_{h}\right) \leq 2 \theta$ and $\angle\left(O_{j}, O_{h}\right)>2 \theta$. For the first term, $\frac{3}{2} \frac{r_{y}^{2}(n) \phi_{y} \theta}{2 \pi}$ is the average area sensors might locate to $\theta$-view cover $O_{j}$ or $O_{h}$. Since the overlapping area between $O_{j}$ and $O_{h}$ is a random variable, uniformly distributed between 0 and $\theta r^{2}(n)$, the corresponding possible area is also a random variable, uniformly distributed between $\frac{r_{y}^{2}(n) \phi_{y} \theta}{2 \pi}$ and $2 \frac{r_{y}^{2}(n) \phi_{y} \theta}{2 \pi}$, so that its expectation is $\frac{3}{2} \frac{r_{y}^{2}(n) \phi_{y} \theta}{2 \pi}$. We can analyze the second term similarly.

Then with Lemma (1) and Eq. (4), we obtain that

$\sum_{P_{i} \in \mathbb{M}} \sum_{O_{j}, O_{h} \in \mathbb{K}}^{O_{j} \neq O_{h}} \mathbb{P}\left(\left\{O_{j}\right.\right.$ and $O_{h}$ of $P_{i}$ is uncovered $) \sim \frac{\theta}{\pi} e^{-3 \omega}$. 
The proof is available in technical report [25].

Since we consider the asymptotic coverage problem, which means that the total number of cameras $n$ approaches to infinity. Then for any fixed $\omega$, we obtain that

$$
\liminf _{n \rightarrow \infty} P_{f-r w}(n, r(n)) \geq e^{-2 \omega}-\frac{\theta}{\pi} e^{-3 \omega} .
$$

Now we consider the case when $\omega$ is a function of $n$ with $\omega=\lim _{n \rightarrow \infty} \omega(n)$, which indicates that $\omega(n)<\omega+\delta$ for any $\delta>0$, for all $n>N_{\delta}$. Since $P_{f-r w}(n, r(n))$ is monotonously decreasing in $r_{\text {stat }}$ and thus in $\omega$, we have

$$
\liminf _{n \rightarrow \infty} P_{f-r w}(n, r(n)) \geq e^{-2(\omega+\delta)}-\frac{\theta}{\pi} e^{-3(\omega+\delta)},
$$

for all $n>N_{\delta}$.

From Proposition 1, we know that $P_{f-r w}(n, r(n))$ is bounded away from zero. Combined with the definition of ESR for static model, we know that $R_{\text {stat }} \geq \sqrt{\frac{2(\log n+\log (\log n))}{n \theta}}$ is necessary to achieve the full view coverage of $\mathbb{M}$.

\section{B. Sufficient Condition of Theorem 2}

First, we obtain the following proposition.

Proposition 2. In CSN, if $n$ sensors are randomly and uniformly deployed in an unit square, and $r_{\text {stat }}=c R_{\text {stat }}(n)$ where $c>1$, then

$$
\liminf _{n \rightarrow \infty} \mathbb{P}(\widehat{\mathcal{H}})=0 .
$$

where $\widehat{\mathcal{H}}$ denotes the event that the operational region is not full view covered as defined in Section II.

proof: We suppose that $r=c R_{\text {stat }}(n)$ where $c>1$, and use $F_{i}$ to denote the event that a point $P_{i}$ is not full view covered, $F_{i, j}$ to denote that $O_{j}$ of $P_{i}$ is not $\theta$-view covered. Then, it suffices to prove that

$$
\lim _{n \rightarrow \infty} P\left(\bigcup_{i=1}^{m} F_{i}\right)=0 .
$$

Deriving its upper bound, we have

$$
P\left(\bigcup_{i=1}^{m} F_{i}\right) \leq \sum_{i=1}^{m} P\left(F_{i}\right) \leq \sum_{i=1}^{m} \sum_{j=1}^{k} P\left(F_{i, j}\right) .
$$

We use similar method in the necessary condition part to calculate $P\left(F_{i, j}\right)$ as follows

$$
\begin{aligned}
\mathbb{P}(\widehat{\mathcal{H}}) & =\mathbb{P}\left(\bigcup_{i=1}^{m} \mathcal{F}_{i}\right) \\
& \leq(n \log n)^{2} \prod_{y=1}^{u}\left(1-\frac{r_{y}^{2} \phi_{y} \theta}{2 \pi}\right) \\
& \sim(n \log n)^{2} e^{-n \theta\left(r_{\text {stat }}\right)^{2}} \\
& =\frac{1}{(n \log n)^{2 c^{2}-2}} \rightarrow 0,
\end{aligned}
$$

for any $c>1$.

Then from Proposition 2 and the definition of critical ESR for static model, we know that $R_{\text {stat }} \geq \sqrt{\frac{2(\log n+\log (\log n))}{n \theta}}$ is sufficient to achieve the full view coverage of $\mathbb{M}$.

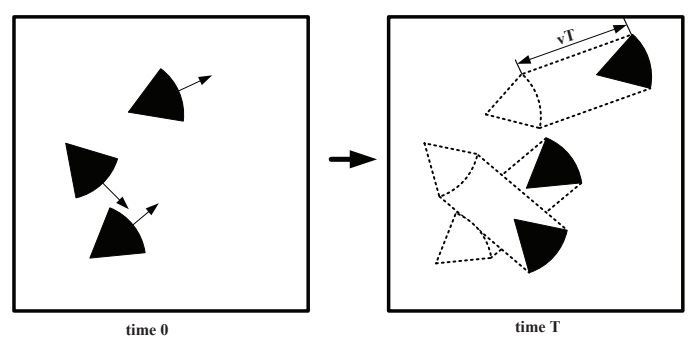

Fig. 3. Full view coverage of CSNs under 2-dimensional random walk: the left figure depicts the initial network configuration at time 0 and the right illustrates the effect of sensor mobility during time interval $[0, T)$. The solid disks constitute the area being covered at the given time instant, and the union of the region inside the dotted line and the solid disks represents the area being covered during the time interval

\section{Critical ESR for Full View Coverage of the Operational Range}

Until now we have already proved that $R_{\text {stat }} \geq$ $\sqrt{\frac{2(\log n+\log (\log n))}{n \theta}}$ is the critical condition to achieve full view coverage for dense grid $\mathbb{M}$. Referring to Lemma 3.1 in [2], as well as Theorem 1 in this paper and using similar approach as THEOREM 4.1 in [2], the density of the dense grid $m=n \log n$ and the density of the orientation set $k=n \log n$ are sufficiently large to evaluate the full view coverage of the whole area. Therefore, Theorem 2 follows.

\section{The CRitical Sensing Range For Mobile CAMERA SENSOR NETWORKS}

In this section, we investigate full view coverage problem for camera sensor networks under uniform deployment under three different mobile patterns, namely, 2-dimensional random walk mobility model, 1-dimensional random walk mobility model and random rotating mobility model.

\section{A. Critical ESR Under 2-Dimensional Random Walk Mobility Model}

We investigate full view coverage in one time slot under 2-Dimensional Random Walk Mobility Model, and Figure 3 illustrates the effect of random walk mobility of the sensor on area coverage. We will first analyze full view coverage for dense grid $\mathbb{M}$, and then expand it to the whole area.

Theorem 3. Under the uniform deployment with 2dimensional random walk mobility model, the critical ESR for mobile heterogenous CSNs to achieve asymptotic full view coverage is

$$
R_{2 . r . w}(n)=\left\{\begin{array}{ll}
\frac{\log n+\log \log n}{2 n T \sin \theta} & \text { if } \theta<\frac{\pi}{2} \\
\frac{\log n+\log \log n}{2 n T v} & \text { if } \theta \geq \frac{\pi}{2}
\end{array} .\right.
$$

We will mainly focus on the condition that $\theta<\frac{\pi}{2}$, and it is similar when $\theta \geq \frac{\pi}{2}$. 
1) Failure Probability of an Orientation in $\mathbb{K}:$ Let $\mathcal{F}_{i, j}$ denote the event that orientation $O_{j}$ of point $P_{i}$ is not $\theta$ viewed covered during the time slot $\tau$, and $\mathbb{P}\left(\mathcal{F}_{i, j}\right)$ denote the corresponding probability. We use $\mathbb{P}_{i, j, S_{y}}$ to represent that $O_{j}$ of point $P_{i}$ is $\theta$-viewed covered by sensor $\mathrm{S}$ in group $G_{y}$. Then we obtain

$$
\mathbb{P}_{i, j, S_{y}}=\left((\theta+\alpha) r_{y}^{2}(n)+2 v \operatorname{Tr}_{y}(n) \sin \theta\right) \frac{\phi_{y}}{2 \pi},
$$

where $\alpha r_{y}^{2}(n)$ represents the additional area due to rotation.

Then, $\mathbb{P}\left(\mathcal{F}_{i, j}\right)$ can be easily calculated.

2) Necessary ESR for Full View Coverage of the Dense Grid: Here, we use $\widehat{\mathcal{H}}^{\tau}$ denote the event that the dense grid $\mathbb{M}$ is not fully full view covered in the time slot $\tau$, and present the following proposition regarding the necessary condition.

Proposition 3. In the mobile heterogeneous CSN with 2dimensional random walk mobility model, if $r_{2 . r . w .}=$ $\frac{\log n+\log \log n+\xi(n)}{2 n T v \sin \theta}$ and the density of the dense grid $\mathbb{M}$ is $m=$ $n \log n$, the density of the orientation set $\mathbb{K}$ is $k=n \log n$, then

$$
\liminf _{n \rightarrow \infty} \mathbb{P}_{\tau}\left(\widehat{\mathcal{H}^{\tau}}\right) \geq e^{-2 \xi}-\frac{\theta}{\pi} e^{-3 \xi},
$$

where $\xi=\lim _{n \rightarrow \infty} \xi(n)$.

proof: Similarly to the proof of Proposition 1, we first study the case where $r_{2 . r . w .}=\frac{\log n+\log \log n+\xi}{2 n T v \sin \theta}$, for a fix $\xi$.

$$
\begin{aligned}
\mathbb{P}_{\tau}\left(\widehat{\mathcal{H}^{\tau}}\right) & \geq \sum_{P_{i} \in \mathbb{M}} \sum_{O_{j} \in \mathbb{K}} \mathbb{P}_{\tau}\left(\left\{O_{j} \text { of } P_{i} \text { is uncovered }\right\}\right) \\
& -\sum_{P_{i} \in \mathbb{M}} \sum_{O_{j}, O_{h} \in \mathbb{K}}^{O_{j} \neq O_{h}} \mathbb{P}_{\tau}\left(\left\{O_{j} \text { and } O_{h} \text { of } P_{i} \text { are uncovered }\right) .\right.
\end{aligned}
$$

We could bound the first term of R.H.S of Eq. (11),

$$
\sum_{P_{i} \in \mathbb{M}} \sum_{O_{j} \in \mathbb{K}} \mathbb{P}_{\tau}\left(\left\{O_{j} \text { of } P_{i} \text { is uncovered }\right\}\right) \sim e^{-2 \xi},
$$

Similarly, we bound the second term

$$
\sum_{P_{i} \in \mathbb{M}} \sum_{O_{j}, O_{h} \in \mathbb{K}}^{O_{j} \neq O_{h}} \mathbb{P}_{\tau}\left(\left\{O_{j} \text { and } O_{h} \text { of } P_{i} \text { are uncovered }\right) \sim \frac{\theta}{\pi} e^{-3 \xi} .\right.
$$

The proof is available in technical report [25].

Then we have

$$
\liminf _{n \rightarrow \infty} \mathbb{P}_{\tau}\left(\widehat{\mathcal{H}^{\tau}}\right) \geq e^{-2 \xi}-\frac{\theta}{\pi} e^{-3 \xi},
$$

Taking into account that $\xi$ is a function of $n$, the conclusion still holds.

According to Proposition 8, we know that $R_{2 . r . w .} \geq$ $\frac{\log n+\log \log n+\xi(n)}{2 n T v \sin \theta}$ is necessary to achieve the full view coverage of $\mathbb{M}$.
3) Sufficient ESR for Full View Coverage of the Dense Grid: First, we obtain the following proposition.

Proposition 4. In CSN, if $n$ sensors are randomly and uniformly deployed in an unit square, and $r_{2 . r . w .}=c R_{2 . r . w .}(n)$ where $c>1$, then

$$
\liminf _{n \rightarrow \infty} \mathbb{P}_{\tau}\left(\widehat{\mathcal{H}_{\tau}}\right)=0
$$

proof: Using similar approach as Proposition 2, we can proof this proposition.

From Proposition 4 and the definition of critical ESR for 2-dimensional random walk mobility model, we know that $R_{2 . r . w .} \geq \frac{\log n+\log \log n+\xi(n)}{2 n T v \sin \theta}$ is sufficient to achieve the full view coverage of $\mathbb{M}$.

4) Critical ESR for Full View Coverage of the Operational Range: Similar to the analysis in the static model, Theorem 3 follows.

\section{B. Critical ESR Under 1-Dimensional Random Walk Mobility Model}

1) Failure Probability of an Orientation in $\mathbb{K}$ : Similarly, let $\mathcal{F}_{i, j}$ denote the event that orientation $O_{j}$ of point $P_{i}$ is not $\theta$-viewed covered during the time slot $\tau$, and $\mathbb{P}\left(\mathcal{F}_{i, j}\right)$ denote the corresponding probability. We use $\mathbb{P}_{i, j, S_{y}}$ to represent that $O_{j}$ of point $P_{i}$ is $\theta$-viewed covered by sensor $\mathrm{S}$ in group $G_{y}$.

From Wang in [21], we know that for 1-dimensional random walk mobility model, the probability that $S$ falls in the circle around of $P_{i}$, with radius $r_{y}$ is $\mathbb{P}_{i, S}=\frac{4}{3} r_{y}$. Clearly $\mathbb{P}\left(S\right.$ falls in cirle around $\left.P_{i}\right)=\mathbb{P}_{i, S}$. Then we obtain

$$
\begin{aligned}
\mathbb{P}_{i, j, S_{y}} & =\mathbb{P}\left(\mathrm{S} \text { falls in } T_{j}\right) \times \mathbb{P}(\mathrm{S} \text { has proper orientation }) \\
& =\mathbb{P}\left(\mathrm{S} \text { falls in the cirle around } P_{i}\right) \times \frac{2 \theta}{2 \pi} \times \frac{\phi_{y}}{2 \pi} \\
& =\frac{\theta \phi_{y}}{2 \pi^{2}} \mathbb{P}_{i, S}=\frac{2 \theta \phi_{y} r_{y}(n)}{3 \pi^{2}} .
\end{aligned}
$$

Then, $\mathbb{P}\left(\mathcal{F}_{i, j}\right)$ can be easily calculated.

2) Necessary ESR for Full View Coverage of the Dense Grid: Here, we use $\widehat{\mathcal{H}^{\tau}}$ denote the event that the dense grid $\mathbb{M}$ is not fully full view covered in the time slot $\tau$, and have the following technical lemma.

Lemma 2. If $r_{1 . r . w .}=\frac{3 \pi(\log n+\log \log n+\xi(n))}{2 \theta n}$, and $m(n)=$ $n \log n, k(n)=n \log n$, for fixed $\gamma<1$,

$$
m k \prod_{y=1}^{u}\left(1-\frac{2 \theta \phi_{y} r_{y}(n)}{3 \pi^{2}}\right)^{c_{y} n} \geq \gamma e^{-\xi},
$$

holds for all sufficient large $n$.

proof: Using the same approaching for Lemma 1

Now, we present the following proposition regarding the necessary condition.

Proposition 5. In the mobile heterogeneous CSN with 1dimensional random walk mobility model, if $r_{1 . r . w .}=$ $\frac{3 \pi(\log n+\log \log n+\xi(n))}{2 \theta n}$ and the density of the dense grid $\mathbb{M}$ 
is $m=n \log n$, the density of the orientation set $\mathbb{K}$ is $k=n \log n$, then

$$
\liminf _{n \rightarrow \infty} \mathbb{P}_{\tau}\left(\widehat{\mathcal{H}^{\tau}}\right) \geq \gamma e^{-\xi}-\frac{\theta}{\pi} e^{-4 \xi},
$$

where $\xi=\lim _{n \rightarrow \infty} \xi(n)$.

proof: Similar to the proof of Proposition 1, we first study the case where $r_{1 . r . w .}=\frac{3 \pi(\log n+\log \log n+\xi(n))}{2 \theta n}$, for a fix $\xi$.

$$
\begin{aligned}
\mathbb{P}_{\tau}\left(\widehat{\mathcal{H}^{\tau}}\right) & \geq \sum_{P_{i} \in \mathbb{M}} \sum_{O_{j} \in \mathbb{K}} \mathbb{P}_{\tau}\left(\left\{O_{j} \text { of } P_{i} \text { is uncovered }\right\}\right) \\
& -\sum_{P_{i} \in \mathbb{M}} \sum_{O_{j}, O_{h} \in \mathbb{K}}^{O_{j} \neq O_{h}} \mathbb{P}_{\tau}\left(\left\{O_{j} \text { and } O_{h} \text { of } P_{i} \text { are uncovered }\right) .\right.
\end{aligned}
$$

And we calculate that

$\mathbb{P}_{\tau}\left(\left\{O_{j}\right.\right.$ of $P_{i}$ is uncovered $\left.\}\right)=\prod_{y=1}^{u}\left(1-\frac{2 \theta \phi_{y} r_{y}(n)}{3 \pi^{2}}\right)^{c_{y} n}$.

Using Lemma 2, we bound the first term of R.H.S of Eq. (18),

$$
\sum_{P_{i} \in \mathbb{M}} \sum_{O_{j} \in \mathbb{K}} \mathbb{P}_{\tau}\left(\left\{O_{j} \text { of } P_{i} \text { is uncovered }\right\}\right) \geq \gamma e^{-\xi},
$$

for any $\gamma>1$ and all $n>N_{\xi}$.

Then we bound the second term

$$
\sum_{P_{i} \in \mathbb{M}} \sum_{O_{j}, O_{h} \in \mathbb{K}}^{O_{j} \neq O_{h}} \mathbb{P}_{\tau}\left(\left\{O_{j} \text { and } O_{h} \text { of } P_{i} \text { are uncovered }\right) \sim \frac{\theta}{\pi} e^{-3 \omega} .\right.
$$

The proof is available in technical report [25].

Then we have

$$
\mathbb{P}_{\tau}\left(\widehat{\mathcal{H}^{\tau}}\right) \geq \gamma e^{-\xi}-\frac{\theta}{\pi} e^{-3 \xi}
$$

Taking into account that $\xi$ is a function of $n$, the conclusion still holds.

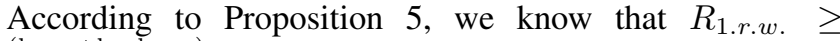
$\frac{3 \pi(\log n+\log \log n)}{2 \theta n}$ is necessary to achieve the full view coverage of $\mathbb{M}$.

3) Sufficient ESR for Full View Coverage of the Dense Grid: First, we obtain the following proposition.

Proposition 6. In CSN, if $n$ sensors are randomly and uniformly deployed in an unit square, and $r_{1 . r . w .}=c R_{1 . r . w .}(n)$ where $c>1$, then

$$
\liminf _{n \rightarrow \infty} \mathbb{P}_{\tau}\left(\widehat{\mathcal{H}}_{\tau}\right)=0
$$

proof: Using similar approach as Proposition 2, this proposition can be proven.

From Proposition 5 and the definition of critical ESR for 1-dimensional random walk mobility model, we know that $R_{1 . r . w .} \geq \frac{3 \pi(\log n+\log \log n)}{2 \theta n}$ is sufficient to achieve the full view coverage of $\mathbb{M}$.

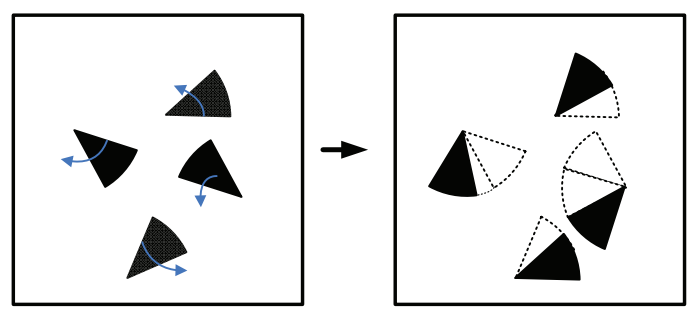

Fig. 4. Full view coverage of CSNs under random rotating walk: the left figure depicts the initial network configuration at the beginning of one time slot, and the right figure illustrates the effect of sensor mobility in this time slot. The solid disks constitute the area being covered at the given time instant, and the union of the region inside the dotted line and the solid disks represents the area being covered in this slot.

4) Critical ESR for Full View Coverage of the Operational Range: Similar to the analysis in the static model, we can reach the following theorem.

Theorem 4. Under the uniform deployment with 1dimensional random walk mobility model, the critical ESR for mobile heterogenous CSNs to achieve asymptotic full view coverage is $R_{1 . r . w .}(n)=\frac{3 \pi(\log n+\log \log n)}{2 \theta n}$.

\section{Critical ESR Under Random Rotating Mobility Model}

We investigate the situation in one time slot under the random rotating mobility pattern. Figure 4 illustrates the effect of random rotating mobility of the sensor on area coverage. We still first analyze the full view coverage for dense grid $\mathbb{M}$, and then expand it to the whole area.

1) Failure Probability of an Orientation in $\mathbb{K}$ : Similarly, $\mathcal{F}_{i, j}$ denotes the event that orientation $O_{j}$ of point $P_{i}$ is not $\theta$-viewed covered, and $\mathbb{P}\left(\mathcal{F}_{i, j}\right)$ denotes the corresponding probability. $\mathbb{P}_{i, j, S_{y}}$ is the same as that under 2-dimensional random walk mobility model.

Then we can obtain

$$
\begin{aligned}
\mathbb{P}_{i, j, S_{y}} & =\mathbb{P}\left(\mathrm{S} \text { falls in } T_{j}\right) \times \mathbb{P}(\mathrm{S} \text { has proper orientation }) \\
& =\pi r_{y}^{2}(n) \times \frac{2 \theta}{2 \pi} \times \mathbb{P}(\mathrm{S}),
\end{aligned}
$$

we could calculate that $\mathbb{P}(S)=\frac{1}{2}\left(1+\frac{\phi_{y}}{\pi}-\frac{\phi_{y}^{2}}{4 \pi^{2}}\right)$. The proof is available in technical report [25].

Then, $\mathbb{P}\left(\mathcal{F}_{i, j}\right)$ can be easily calculated.

2) Necessary ESR for Full View Coverage of the Dense Grid: Here, we use $\widehat{\mathcal{H}^{\tau}}$ denote the event that the dense grid $\mathbb{M}$ is not fully full view covered in the time slot $\tau$. We have the following lemma.

Lemma 3. If $r_{r . r .}=\sqrt{\frac{4(\log n+\log (\log n)+\xi(n))}{n \theta}}$, and $m(n)=$ $n \log n, k(n)=n \log n$, for fixed $\gamma<1$,

$$
m k \prod_{y=1}^{u}\left[1-\frac{\theta}{2}\left(1+\frac{\phi}{\pi}-\frac{\phi^{2}}{4 \pi^{2}}\right) r_{y}^{2}(n)\right]^{c_{y} n} \geq \gamma e^{-\xi},
$$

holds for all sufficient large $n$.

proof: Using the same approaching for Lemma 1. 
Now, we present the following proposition regarding the necessary condition.

Proposition 7. In the mobile heterogeneous CSN with random rotating mobility model, if $r_{r . r .}=\sqrt{\frac{4(\log n+\log (\log n)+\xi(n))}{n \theta}}$ and the density of the dense grid $\mathbb{M}$ is $m=n \log n$, the density of the orientation set $\mathbb{K}$ is $k=n \log n$, then

$$
\liminf _{n \rightarrow \infty} \mathbb{P}_{\tau}\left(\widehat{\mathcal{H}^{\tau}}\right) \geq e^{-\xi}-e^{-4 \xi},
$$

where $\xi=\lim _{n \rightarrow \infty} \xi(n)$.

proof: We use similar approaching as Proposition 1, and only present the main steps here. Firstly, we study the case where $r_{r . r .}=\sqrt{\frac{4(\log n+\log (\log n)+\xi)}{n \theta}}$, for a fix $\xi$.

$$
\begin{aligned}
\mathbb{P}_{\tau}\left(\widehat{\mathcal{H}^{\tau}}\right) & \geq \sum_{P_{i} \in \mathbb{M}} \sum_{O_{j} \in \mathbb{K}} \mathbb{P}_{\tau}\left(\left\{O_{j} \text { of } P_{i} \text { is uncovered }\right\}\right) \\
& -\sum_{P_{i} \in \mathbb{M}} \sum_{O_{j}, O_{h} \in \mathbb{K}}^{O_{j} \neq O_{h}} \mathbb{P}_{\tau}\left(\left\{O_{j} \text { and } O_{h} \text { of } P_{i} \text { are uncovered }\right)\right.
\end{aligned}
$$

Then we bound the two terms on the right correspondingly, and have

$$
\mathbb{P}_{\tau}\left(\widehat{\mathcal{H}^{\tau}}\right) \geq \gamma e^{-\xi}-e^{-4 \xi} .
$$

Furthermore, the result holds for when $\xi$ changes, thus we finish the necessary part.

3) Sufficient ESR for Full View Coverage of the Dense Grid:: Similarly, we obtain the following proposition.

Proposition 8. In CSN, if $n$ sensors are randomly and uniformly deployed in an unit square, and $r_{r . r .}=c R_{r . r .}(n)$ where $c>1$, then

$$
\liminf _{n \rightarrow \infty} \mathbb{P}_{\tau}\left(\widehat{\mathcal{H}_{\tau}}\right)=0
$$

proof: Using same technique as Proposition 2, we have $R_{\text {r.r. }} \geq \sqrt{\frac{4(\log n+\log (\log n))}{n \theta}}$ is sufficient to achieve the full view coverage of $\mathbb{M}$.

4) Critical ESR for Full View Coverage of the Operational Range: Similar to the analysis in the static model, we can reach the following theorem.

Theorem 5. Under the uniform deployment with random rotating mobility model, the critical ESR for mobile heterogenous CSNs to achieve asymptotic full view coverage is $R_{\text {r.r. }}(n)=\sqrt{\frac{4(\log n+\log (\log n))}{n \theta}}$.

\section{Performance Evaluation}

\section{A. Impact of Mobility on Sensing Energy Consumption}

We consider the impact of mobility here. Sensors are considered to have critical ESR, with radius $r_{y}=r_{i}, i=$ stat, 2.r.w., 1.r.w., r.r., under static, 2-dimensional random walk, 1-dimensional random walk, and random rotating correspondingly. As we just convert the value of the angle of each sensor to the weight of its radius when we derive the critical ESR, the sensors can be viewed as omnidirectional traditional sensors and we here use the area the sensor covers to represent the sensing energy consumption of it. Taking static model as a baseline, we have the following results

$$
\begin{gathered}
\bar{E}_{2 . r . w .}=\bar{E}_{1 . r . w .}=\Theta\left(\frac{\log n+\log \log n}{n}\right) \times \bar{E}_{\text {stat }}, \\
\bar{E}_{\text {r.r. }}=\bar{E}_{\text {stat }},
\end{gathered}
$$

which indicates that compared with static model, both the 2dimensional random walk mobility model and 1-dimensional random walk mobility model can decrease the energy consumption in CSNs. And this improvement sacrifices the delay upper bounded by $\Theta(1)$ as the movement is divided into time slots. This is actually a tradeoff between energy consumption and the delay.

However, for random rotating mobility, the energy consumption is the same as when sensors are stationary, but it still causes a delay upper bounded by $\Theta(1)$, due to the division of the time slots. Furthermore, this results in much more energy consumption for movement. Thus, the movements like random rotating should be avoided for full view coverage.

\section{B. Impact of Parameter $n$ and $\theta$}

Here we analyze the influence of $n$ and $\theta$ on the critical equivalent sensing range denoted by $R(n)$.

For Figure 5, when $n$ is fixed, $R(n)$ becomes larger, as $\theta$ decreases for all the static and mobility patterns we have discussed. So we need sensors of larger sensing range when a better view of object's face is required. It is obvious since larger sensing region render more sensors to cover a certain object, thus having more chance to catch its frontal image. When it is large enough (like $n=4000,5000$ in Figure 5 as an example), $n$ ail not further influence the network performance. This fact corresponds with the instinct that when there are plenty of sensors in the network, adding more sensors will not further reduce the critical equivalent sensing range. Furthermore, as shown in Figure 5, changing $n$ will lead to greater change of $R(n)$ for smaller effective angle $\theta$, but $n$ will has little influence on $R(n)$ when $\theta$ goes to $\pi$. We can analyze Figure 6 similarly.

\section{CONCLUSION}

Coverage property of camera sensor networks is a fundamental issue, among which full view coverage draws lots of attention due to its emphasis on capturing the objects' face. In this paper, we study the static and mobile models for Camera Sensor Networks. We mainly focus on heterogeneous camera network and define a parameter named Equivalent Sensing Range to model it. Furthermore, we derive the critical sensing range for full view coverage under static model, 2-dimensional random walk mobility model, 1-dimensional random walk mobility model and random rotating model. These formulations not only provide an asymptotic description of the critical power needed to maintain the full view coverage, 


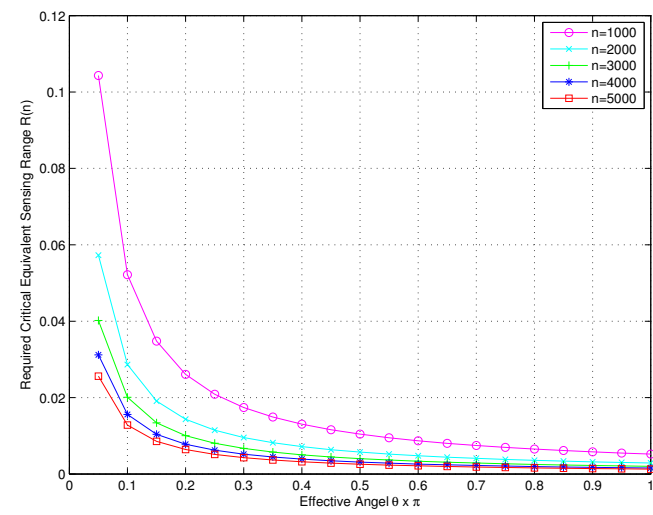

Fig. 5. Relationship between critical ESR $R(n)$ and $\theta$, when $n$ changes accordingly under 1-dimensional random walk mobility model

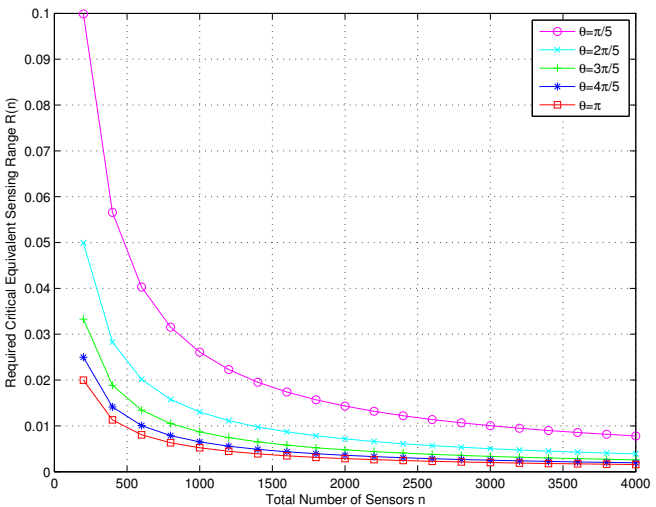

Fig. 6. Relationship between critical ESR $R(n)$ and $n$, when $\theta$ changes accordingly under 1-dimensional random walk mobility model

but also help to identify the impact of mobility on the network. Our results offer some valuable insights for the analysis and design of camera sensor networks, especially for high quality service. There are some interesting topics for future work. First, we would like to investigate the situation when sensors can cooperate with each other, and exchange their orientation and location information. Besides it's interesting to consider that there are obstacles in CSNs.

\section{ACKNOWLEDGE}

This work is supported by NSF China (No. 61325012, 61271219, 61221001, 61102052); Shanghai Basic Research Key Project (No. 13510711300, 12JC1405200, 11JC1405100); China Ministry of Education New Century Excellent Talent (No. NCET-10-0580); National Key Project of China (No. 2012ZX03001009); Doctoral Fund of Ministry of Education of China (No. 13005160); SEU SKL project (No. 2012D13).

\section{REFERENCES}

[1] I. F. Akyildiz, T. Melodia, and K. R. Chowdhury, "A survey on wireless multimedia sensor networks," in Comput. Netw., 51(4): 921-960, 2007.
[2] S. Kumar, T. H. Lai and J. Balogh, "On $k$-coverage in a mostly sleeping sensor networks," in Proc. of ACM MobiCom 2004, Philadelphia, Pennsylvania, USA, Sept., 2004.

[3] S. Shakkottai, R. Srikant, and N. Shroff, "Unreliable Sensor Grids: Coverage, Connectivity and Diameter," in Proc. of IEEE INFOCOM 2003, San Francisco, USA, Mar., 2003.

[4] A. Chen, S. Kumar, and T. H. Lai, "Designing localized algorithms for barrier coverage," in Proc. of ACM MobiCom 2007, Montreal, QC, Canada, Sept., 2007.

[5] A. Saipulla, C. Westphal, B. Liu, and J. Wang, "Barrier coverage of linebased deployed wireless sensor networks," in Proc. of IEEE INFOCOM 2009, Rio de Janeiro, Brazil, Apr., 2009.

[6] B. Liu, O. Dousse, J. Wang, A. Saipulla, "Strong barrier coverage of wireless sensor networks," in Proc. of ACM MobiHoc 2008, Hong Kong SAR, China, May, 2008.

[7] X. Bai, Z. Yun, D. Xuan, B. Chen and W. Zhao, "Optimal multiplecoverage of sensor networks," in Proc. of IEEE INFOCOM 2011, Shanghai, China, April, 2011.

[8] X. Bai, D. Xuan, Z. Yun, T. H. Lai and W. Jia, "Complete optimal deployment patterns for full-coverage and k-connectivity $(k<6)$ wireless sensor networks," in Proc. of ACM MobiHoc 2008, Hong Kong SAR, China, May, 2008.

[9] X. Bai, S. Kumar, D. Xuan, Z. Yun, and T. H. Lai, "Deploying wireless sensors to achieve both coverage and connectivity," in Proc. of ACM MobiHoc 2006, Florence, Italy, May, 2006.

[10] Y. Wang and G. Cao, "On full-view coverage in camera sensor networks," in Proc. of IEEE INFOCOM 2011, Shanghai, China, April, 2011

[11] V. Blanz, P. Grother, P. J. Phillips and T. Vetter, "Face recognition based on frontal views generated from non-frontal images," in Proc. of IEEE Computer Society Conference on Computer Vision and Pattern Recognition (CVPR 05), San Diego, USA, June, 2005.

[12] Y. Wu, and X. Wang, "Achieving Full View Coverage with RandomlyDeployed Heterogeneous Camera Sensors," in Proc. of IEEE ICDCS 2012, Macau, China, June, 2012.

[13] Y. Wang and G. Cao, "Barrier coverage in camera sensor networks," in Proc. of ACM MobiHoc 2011, Paris, France, May, 2011.

[14] H. Ma, M. Yang, D. Li, Y. Hong, and W. Chen, "Minimum camera barrier coverage in wireless camera sensor networks," in Proc. of IEEE INFOCOM 2012, Orando, Florida, USA, March, 2012.

[15] L. Sun, W. Wang, "Understanding the tempo-spatial limits of information dissemination in multi-channel Cognitive Radio Networks," in Proc. of IEEE INFOCOM 2012, Orando, Florida, USA, March, 2012.

[16] B. Liu, P. Brass, O. Dousse, P. Nain and D. Towsley, "Mobility improves coverage of sensor networks," in Proc. of ACM MobiHoc 2005, UrbanaChampaign, Illinois, USA, May, 2005.

[17] A. Saipulla, B. Liu, G. Xing, X. Fu, J. Wang, "Barrier coverage with sensors of limited mobility," in Proc. ACM MobiHoc 2010, Chicago, Illinois, USA, Sept., 2010.

[18] W. Cheng, M. Li, K. Liu, Y. Liu, X. Li, X. Liao, "Sweep coverage with mobile sensors", in Parallel and Distributed Processing, 2008. IPDPS 2008. IEEE International Symposium on, pp. 1-9. IEEE, 2008.

[19] M. Grossglauser, and D. Tse, "Mobility Increases the Capacity of Ad Hoc Wireless Networks," in IEEE/ACM Transactions on Networking, vol. 10, no. 4, pp. 477-486, August 2002.

[20] S. Čapkun, J. Hubaux and L. Buttyán, "Mobility Helps Security in Ad Hoc Networks," in Proc. ACM MobiHoc 2003, Annapolis, MD, USA, June, 2003.

[21] X. Wang, X. Wang and J. Zhao, "Impact of mobility and heterogeneity on coverage and energy consumption in wireless sensor networks," in Proc. of IEEE ICDCS 2011, Minneapolis, USA, June, 2011.

[22] H. M. Ammari, and J. Giudici. "On the connected k-coverage problem in heterogeneous sensor nets: The curse of randomness and heterogeneity." in Proc. of IEEE ICDCS 2009, Montreal, Quebec, Canada, June, 2009.

[23] M. Cardei, and H. Gupta, "Selection and orientation of directional sensors for coverage maximization," in Proc. of IEEE SECON 2009, Rome, Italy, June, 2009.

[24] Y. Wang, and G. Cao, "Minimizing service delay in directional sensor networks," in Proc. of IEEE INFOCOM 2011, Shanghai, China, April, 2011.

[25] Y. Hu, S. Han, X. Wang, X. Gan, "Critical Sensing Range for Mobile Heterogeneous Camera Sensor Networks, Shanghai Jiao Tong University, Tech. Rep., 2013. [Online]. Available: iwct.sjtu.edu.cn/Personal/xwang8/paper/coverage-camerasensor.pdf. 